\title{
Cascade Neuro-Fuzzy Architecture Based Mobile- Robot Navigation and Obstacle Avoidance in Static and Dynamic Environments
}

\author{
Anish Pandey*, and Kavita Burse \\ Oriental College of Technology, Bhopal, India
}

Received: September 16, 2016; Accepted: September 28, 2016; Published: October 05, 2016

*Corresponding author: Anish Pandey, Oriental College of Technology, Bhopal, Ganga Nagar SEC-2 Mangla Road Bilaspur, Chhattisgarh, India, Tel: +919861932338; E-mail: Anish06353@Gmail.Com

\begin{abstract}
Real-time navigation in the partially unknown environment is an interesting task for mobile robotics. This article presents the cascade neuro-fuzzy (CN-Fuzzy) architecture for intelligent mobile robot navigation and obstacle avoidance in static and dynamic environments. The array of ultrasonic range finder sensors and sharp infrared range sensors are used to read the front, left and right obstacle distances. The cascade neural network is used to train the robot to reach the goal. Its inputs are the different obstacle distance received from the sensors. The output of the neural network is a turning angle between the robot and goal. The fuzzy architecture is integrated with the cascade neural network to control the velocities of the robot. Successful simulation and experimental results verify the effectiveness of the proposed architecture in both static and dynamic environments. Moreover, the proposed CN-Fuzzy architecture gives better results (in terms of path length) as compared to previously developed techniques, which verifies the effectiveness of the proposed architecture.
\end{abstract}

Keywords: Cascade neuro-fuzzy; Fuzzy logic; Neural networks; Mobile robots; Obstacle avoidance; Velocity

\section{Introduction}

The applications of the intelligent robot in many fields such as industry, space, agriculture, defense and transportation, and other social sectors are growing day by day. The mobile robot performs many tasks such as rescue operation, patrolling, underwater exploration, disaster relief and planetary exploration, etc. Therefore, the author is trying to put the effort in the field of the intelligent robot using CN-Fuzzy architecture, which can avoid the obstacle autonomously and reach the goal safely in the given environment. Autonomous mobile robot navigation is one of the challenging tasks for any soft computing techniques. Fuzzy logic and neural network have been widely used for mobile robot navigation and control because these methods are capable of handling the system uncertainty. Generally, the fuzzy logic is the combination of fuzzy rules and membership functions (inputs and outputs), which are constructed by human knowledge. And the neural network may be applied to a linear or nonlinear system, which can solve the real system problems using empirical data set (experimental or predicted). The neural network with fuzzy logic [1] improves the decision speed of the mobile robot for target seeking and obstacle avoidance.

Target seeking and obstacle avoidance are the two important tasks for any mobile robot in the environment. Godjevac and Steele [2] have integrated the Takagi-Sugeno type fuzzy controller and Radial Basis Function Neural Network (RBFNN) to solve the mobile robot path planning. Where the fuzzy logic is used to handle the uncertainty of the environment, and the neural network is used to tune the parameters of membership functions. Rai and Rai, [3] have designed the Arduino UNO microcontroller-based DC motor speed control system using multilayer neural network and Proportional Integral Derivative (PID) controller. Yang and Meng [4] have applied the biologically inspired neural network to generate a collision-free path in a nonstationary environment. In [5], the authors have designed the Reinforcement Ant Optimized Fuzzy Controller (RAOFC) and applied it for wheeled mobile robot wall-following control under reinforcement learning environments. The inputs of the proposed controller are rangefinding sonar sensors, and the output is a robot steering angle. Algabri, et al. [6] have combined the fuzzy logic with other soft computing techniques such as Genetic Algorithm (GA), Neural Networks (NN), and Particle Swarm Optimization (PSO) to optimize the membership function parameters of the fuzzy controller for improving the navigation performance of the mobile robot. Fuzzy reinforcement learning sensor-based mobile robot navigation has been presented by Beom and Cho [7] for complex environments. In [8], the authors have constructed behaviour-based neuro-fuzzy control architecture for mobile robot navigation in an unstructured environment. Rossomando and Soria, [9] have designed an adaptive neural network PID controller to solve the trajectory tracking control problem of a mobile robot. In [10], the authors have developed a genetic algorithm to choose the best membership parameters from the fuzzy inference system and implemented it to control the steering angle of a mobile robot in the partially unknown environment. In [11], the authors have presented the navigation method of the 
two robots (a leader robot and a follower robot) using Fuzzy Controllers (FC). In [15-16], the authors have designed the sensor based adaptive neuro-fuzzy inference controller for mobile robot navigation and obstacle avoidance in the various environments.

Cascade Neural Network (CNN) is similar to feed Forward Neural Network (FNN). Both neural networks use back propagation algorithm for updating the weights and biases [12]. This article describes the cascade neural network based fuzzy architecture for mobile-robot navigation and obstacle avoidance in static and dynamic environments. The cascade neural network is used to train the robot to reach the goal. Its inputs are different obstacle distance received from the sensors. The output of the neural network is a turning angle between the robot and goal. The fuzzy logic architecture is used to control the right motor velocity and left motor velocity of the mobile robot. In the last two decades, many researchers have implemented different neurofuzzy techniques for solving the navigation problem of the mobile robot. Motivated by the above literature survey, the primary objective of this paper is to improve the navigation accuracy and efficiency of the mobile robot using the cascade neuro-fuzzy controller. The remainder of this article is structured as follows: Section 2 introduces the design and implementation of the CNFuzzy architecture for mobile robot navigation and obstacle avoidance in various environments. Section 3 demonstrates the computer simulation results in different unknown environments. Section 4 describes the simulation result comparison with developed techniques. Section 5 presents the experimental results and discussion for validating the proposed controller. Finally, Section 6 depicts the summary.

\section{Cascade Neuro-Fuzzy (CN-Fuzzy) Architecture}

This section introduces the design and implementation of the CN-Fuzzy architecture for navigation of mobile robot and obstacle avoidance in various environments. The cascade neural network is used to train the robot to reach the goal in the environment, and the fuzzy logic architecture is used to control the right motor velocity and left motor velocity of the mobile robot. Figure 1 shows the proposed architecture of CN-Fuzzy for navigation of mobile robot and obstacle avoidance in unknown environments.

\section{Cascade neural network for goal reaching}

The neural network is one of the important techniques for the mobile robot navigation. In this section, the Cascade Neural Network (CNN) is used to train the robot to reach the goal in the environment. The neural network is the combination of many layers such as input layer, hidden (intermediate) layers, and the output layer; all the layers are connected with each other by the neurons. The CNN is the similar to the feed Forward Neural Network (FNN). Both CNN and FNN use back propagation algorithm for updating the weights and biases. The two back propagation algorithms, namely Levenberg-Marquardt (LM) and Bayesian Regularization (BR) are used to adjust the network weights and biases. Figure 2 illustrates the general structure of a Cascade Neural Network (CNN). In Figure 2, $u, w, b$ and $v$ addresses the input variables, synaptic weights, neuron bias, and output variable, respectively.

The inputs of the CNN are the obstacle distance received from the various sensors, and the output of the CNN is a turning angle between the robot and goal. Table 1 describes the different training patterns for the cascade neural network, which helps the robot to reach the goal in the environment. The proposed CNN uses three inputs, two hidden layers (six and four neurons, respectively) and single output layer for the mobile robot navigation. The CNN has three inputs: F.O.D. (Front Obstacle Distance), L.O.D. (Left Obstacle Distance), and R.O.D. (Right Obstacle Distance), respectively. The output of this CNN is a Turning Angle (T.A.) between the robot and goal. The input and output of the CNN can be written as follows: -

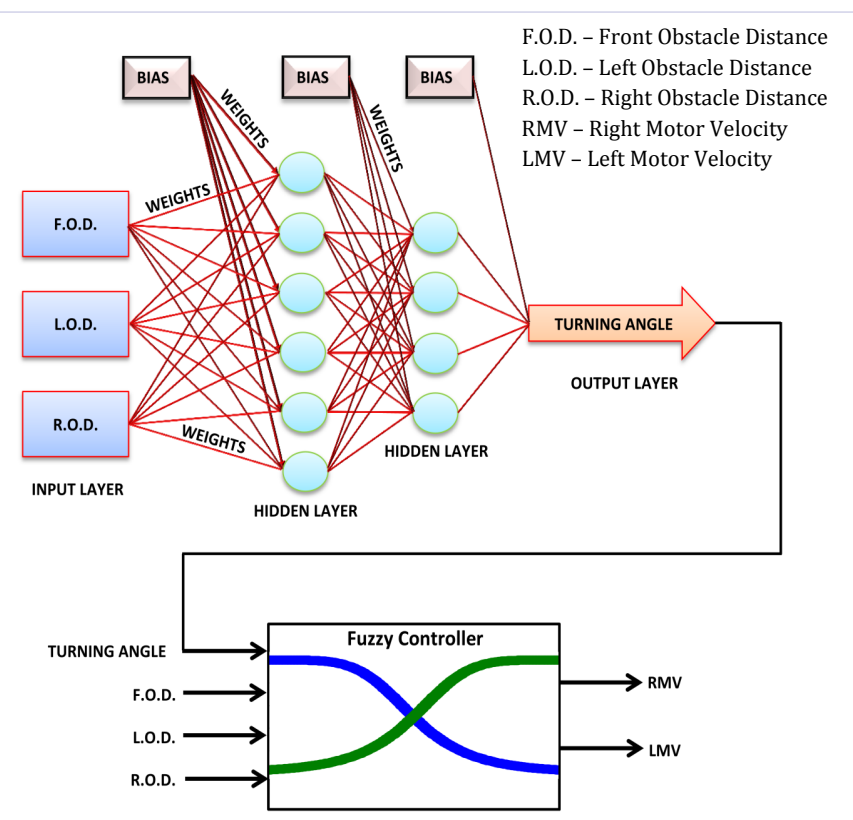

Figure 1: The cascade neuro-fuzzy architecture for navigation of mobile robot and obstacle avoidance in unknown environments

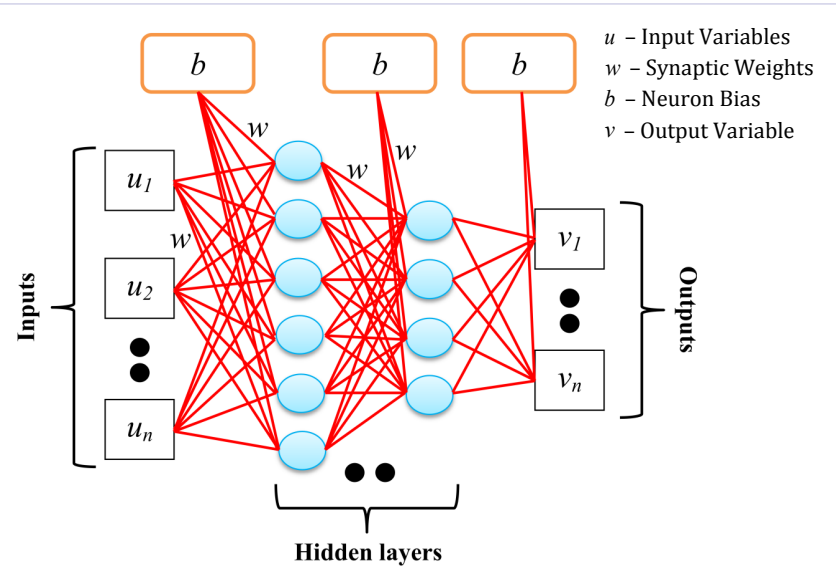

Figure 2: The general structure of the Cascade Neural Network (CNN) 
Where $=1,2$, 3. (Three inputs F.O.D., L.O.D., and R.O.D., respectively)

Input layer (first layer):

$q_{i}^{[1]}=u_{i}$

Two hidden layers (second and third):

$$
q_{t}^{[s]}=\varphi\left(N E T_{t}^{[s]}\right)
$$

$$
N E T_{t}^{[s]}=\sum_{i}\left(w_{t \cdot i}^{[s]} \cdot q_{i}^{[s-1]}+b_{t}^{[s]}\right)
$$

Where $S=2,3$. (Second and third layers)

Output layer (fourth layer):

$$
\begin{aligned}
& v_{(p)}=q^{[4]}=\varphi\left(N E T^{[4]}\right) \\
& N E T^{[4]}=\sum_{i}\left(w_{i}^{[4]} \cdot q_{i}^{[3]}+b_{i}^{[4]}\right)
\end{aligned}
$$

Where $u_{i}$ is the input variables, $v_{(p)}$ is the predicted output variable (turning angle). The $w_{t \cdot i}^{[s]}$ is the synaptic weight on connection joining the $i$ th neuron in the layer $[s-1]$ to the $t$ th neuron in the layer $[s] ; b_{t}^{[s]}$ is a bias of the $t$ th neuron in the layer $[s]$, and $\varphi_{t}(d)$ is the Log-sigmoid transfer function.

$$
\varphi(d)=\frac{1}{(1+\exp (-d))}
$$

The proposed CNN is verified through the mean squared error (MSE) and root mean square error (RMSE) method: -

$$
\operatorname{MSE}(\%)=\left[\sum_{1}^{r}\left(\frac{v_{(a)}-v_{(p)}}{r}\right)^{2}\right] \times 100
$$

Table 1: The different training patterns for mobile robot navigation

\begin{tabular}{|l|l|l|l|l|}
\hline F.O.D. (cm) & $\begin{array}{l}\text { L.O.D. } \\
\text { (cm) }\end{array}$ & $\begin{array}{l}\text { R.o.D. } \\
\text { (cm) }\end{array}$ & $\begin{array}{l}\text { T.A. } \\
\text { (degree) }\end{array}$ & Turning Direction \\
\hline 20 & 115 & 20 & 74.3 & Left \\
\hline 20 & 20 & 150 & -65.9 & Right \\
\hline 125 & 25 & 150 & -70.4 & Right \\
\hline 25 & 75 & 50 & 55 & Left \\
\hline 40 & 120 & 60 & 59.4 & Left \\
\hline 25 & 150 & 100 & 72.8 & Left \\
\hline 25 & 50 & 120 & -22.9 & Right \\
\hline 22 & 25 & 22 & 73.4 & Left \\
\hline 50 & 25 & 25 & 0 & Straight \\
\hline 20 & 27 & 27 & 77 & Left \\
\hline 100 & 28 & 25 & 0 & Straight \\
\hline 25 & 21 & 22 & 77.2 & Left \\
\hline 150 & 25 & 115 & -70.5 & Right \\
\hline 150 & 20 & 25 & 0 & Straight \\
\hline 150 & 100 & 100 & -70.4 & Right \\
\hline
\end{tabular}

$\operatorname{RMSE}(\%)=\sqrt{\frac{1}{r}\left[\sum_{1}^{r}\left(\frac{v_{(a)}-v_{(p)}}{v_{(a)}}\right)^{2}\right]} \times 100$

Where $v_{(a)}$ is the actual output variable, $v_{(p)}$ is the predicted (network) output variable, and $r$ is the number of observations. $u, w, b$, and $v$ addresses the input variables, synaptic weights, neuron bias, and output variable, respectively.

\section{Fuzzy Logic Architecture (FLA) for obstacle avoidance}

This section describes the design of Mamdani-type fuzzy logic [17] architecture for navigation of mobile robot and obstacle avoidance in unknown environments. The Fuzzy Logic Architecture (FLA) is used to control the right motor velocity and left motor velocity of the mobile robot. The proposed FLA has four inputs and two outputs. The FLA receives first three inputs (obstacle distance) from the various sensors of the mobile robot. The first three inputs are denoted by F.O.D., L.O.D., and R.O.D., respectively. The fourth input is the turning angle (goal angle) between the robot and goal, and which is received from the CNN. The outputs of the FLA are the velocities of the motors of robot. The outputs are addressed by RMV (Right Motor Velocity) and LMV (Left Motor Velocity), respectively. The range of first three inputs is divided into two linguistic variables, namely CLOSE and AWAY, respectively, and it is located between $20 \mathrm{~cm}$ to $150 \mathrm{~cm}$. The two linguistic variables NEGATIVE and POSITIVE, respectively, are used for turning angle. The range of outputs is divided into two linguistic variables, namely LOW and HIGH, respectively. The two generalized bell-shaped (Gbell) membership functions are used for inputs and outputs. Figure 3 shows the input and output variables of the FLA. Figure 4 illustrates the fuzzy logic architecture. The fuzzy rule set of the FLA is described in Table 2. The FLA is composed through Mamdani-type fuzzy model in the following form: Rule $_{m}$ : IF $x_{1}$ is $A_{j 1}, x_{2}$ is $A_{j 2}, x_{3}$ is $A_{j 3}$, \& $x_{4}$ is $A_{j 4}$ THEN $y_{1}$ is $B_{j 1}$ \& $y_{2}$ is $B_{j 2}$ (9)

Where $m=1,2,3 \ldots 12$ (twelve rules), the $x_{1}, x_{2}, x_{3}$, and $x_{4}$ are the input variables. Similarly, $y_{1}$ and $y_{2}$ are the output variables. The $A_{j 1}, A_{j 2}, A_{j 3}$, and $A_{j 4}$ are the fuzzy sets of the input variables. Similarly, $B_{j 1}$ and $B_{j 2}$ are the fuzzy sets of the output variables. The $j=1,2$, because each input and output have two Gbell membership functions. The fuzzy set (inputs and outputs) uses the following Gbell membership function: -

$$
\begin{aligned}
& \mu_{j k}\left(x_{k} ; a, b, c\right)=\frac{1}{1+\left|\frac{x_{k}-c_{j k}}{a_{j k}}\right|^{2 b_{j k}}} \\
& \mu_{j l}\left(y_{l} ; a, b, c\right)=\frac{1}{1+\left|\frac{y_{l}-c_{j l}}{a_{j l}}\right|^{2 b_{j l}}}
\end{aligned}
$$


Where $k=1 \ldots 4$ (four inputs), and $l=1,2$ (two outputs). The symbols $a, b$, and $c$ are adjusting parameters of the Gbell membership function; called as the half width, slope control, and centre respectively.

The defuzzification of the output variables $\left(y_{1}\right.$ and $\left.y_{2}\right)$ are accomplished by the weighted average method: -

$$
y_{1}=\frac{\sum_{m=1}^{12}\left(\mu_{j 1}\left(x_{1}\right) \cdot \mu_{j 2}\left(x_{2}\right) \cdot \mu_{j 3}\left(x_{3}\right) \cdot \mu_{j 4}\left(x_{4}\right)\right) \cdot y_{1}}{\sum_{m=1}^{12}\left(\mu_{j 1}\left(x_{1}\right) \cdot \mu_{j 2}\left(x_{2}\right) \cdot \mu_{j 3}\left(x_{3}\right) \cdot \mu_{j 4}\left(x_{4}\right)\right)}
$$

(i)

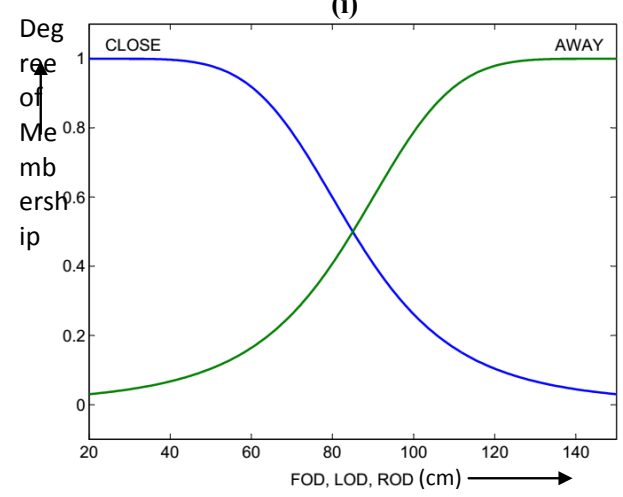

(ii)

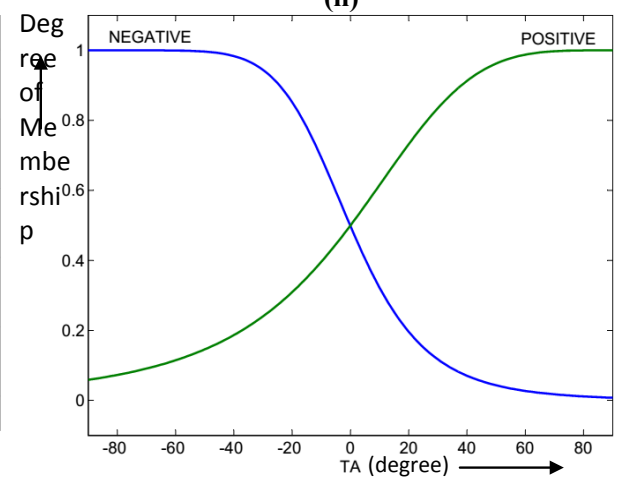

(iii)

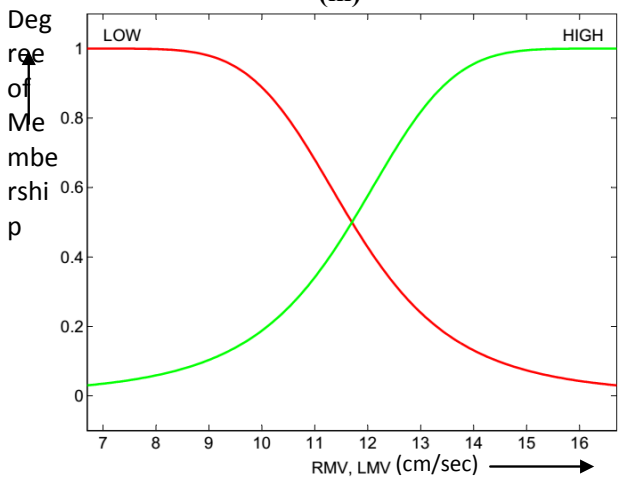

Figure 3:Membership Functions (i), Obstacle Distances (F.O.D., L.O.D. and R.O.D., respectively), (ii) Turning Angle (TA), and (iii) Motor Velocities (Right and Left respectively)

Table 2: Fuzzy rule sets for navigation of mobile robot and obstacle avoidance

\begin{tabular}{|c|c|c|c|c|c|c|}
\hline Fuzzy Rules & F.O.D. (cm) & L.O.D. (cm) & R.O.D. (cm) & T. A. (degree) & $\operatorname{RMV}(\mathrm{cm} / \mathrm{sec})$ & $\operatorname{LMV}(\mathrm{cm} / \mathrm{sec})$ \\
\hline 1 & Away & Away & Away & Positive & High & Low \\
\hline 2 & Away & Away & Away & Negative & Low & High \\
\hline 3 & Close & Close & Close & Negative & Low & High \\
\hline 4 & Close & Close & Close & Positive & High & Low \\
\hline 5 & Away & Close & Away & Negative & Low & High \\
\hline 6 & Away & Away & Close & Positive & High & Low \\
\hline 7 & Close & Away & Away & Negative & Low & High \\
\hline 8 & Close & Away & Away & Positive & High & Low \\
\hline 9 & Close & Close & Away & Negative & Low & High \\
\hline 10 & Close & Away & Close & Positive & High & Low \\
\hline 11 & Away & Close & Close & Positive & High & Low \\
\hline 12 & Away & Close & Close & Negative & Low & High \\
\hline
\end{tabular}




$$
y_{2}=\frac{\sum_{m=1}^{12}\left(\mu_{j 1}\left(x_{1}\right) \cdot \mu_{j 2}\left(x_{2}\right) \cdot \mu_{j 3}\left(x_{3}\right) \cdot \mu_{j 4}\left(x_{4}\right)\right) \cdot y_{2}}{\sum_{m=1}^{12}\left(\mu_{j 1}\left(x_{1}\right) \cdot \mu_{j 2}\left(x_{2}\right) \cdot \mu_{j 3}\left(x_{3}\right) \cdot \mu_{j 4}\left(x_{4}\right)\right)^{1}}
$$

\section{Computer Simulation Results}

This section presents the computer simulation results using CN-Fuzzy architecture in the different unknown static and dynamic environments. The simulations have done using MATLAB

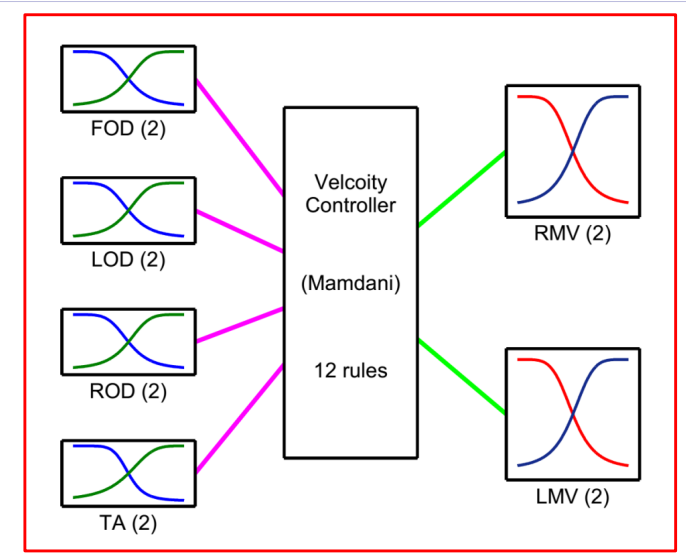

Figure 4: Fuzzy logic architecture

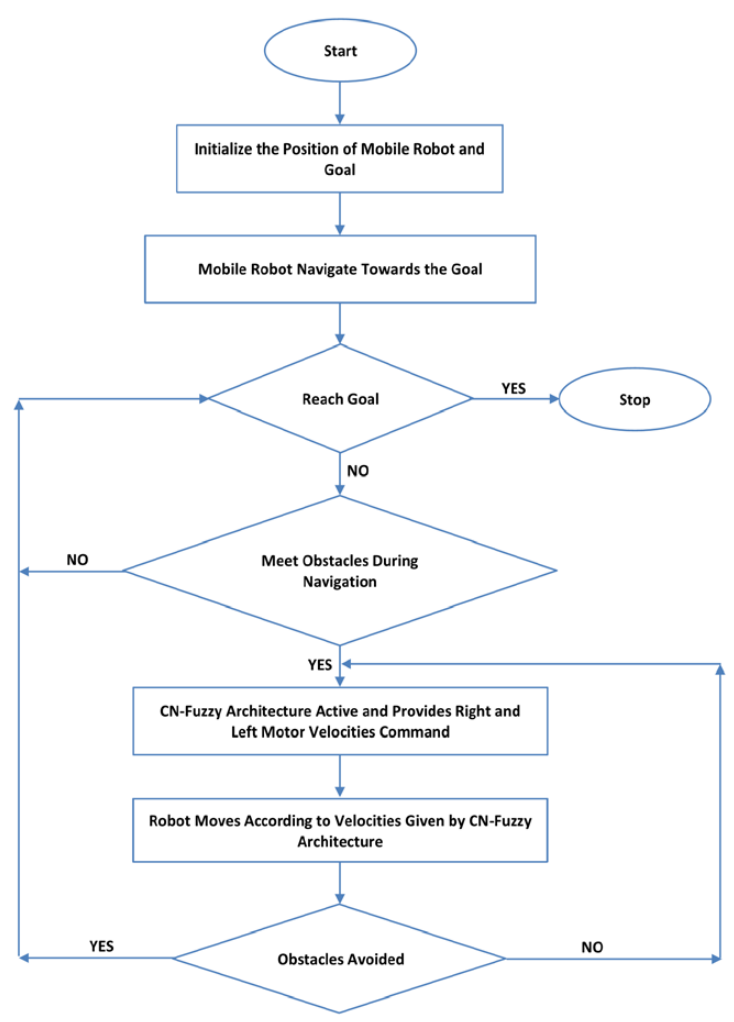

Figure 5: Flowchart of the mobile robot navigation and obstacle avoidance based on CN-Fuzzy architecture

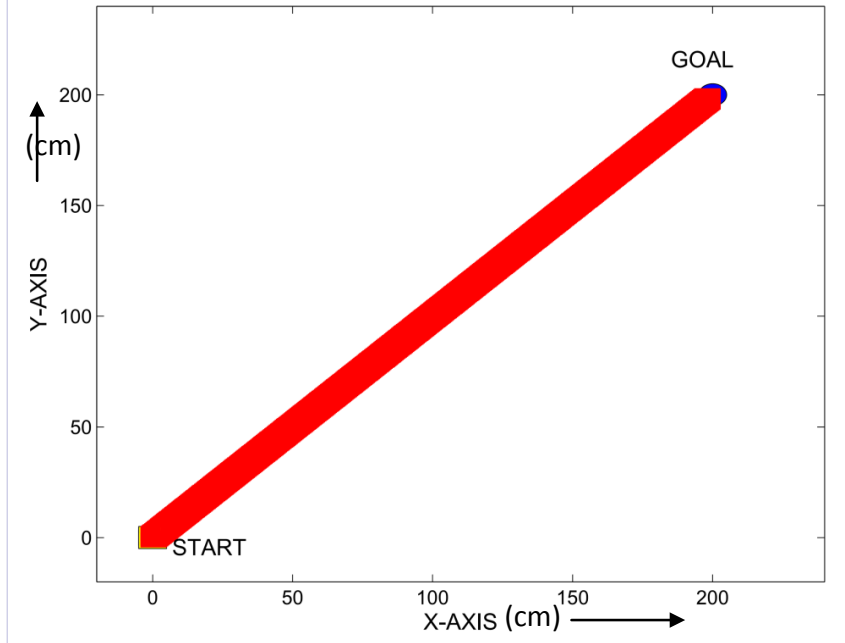

Figure 6: Mobile robot navigation in an environment without obstacle using CN-Fuzzy architecture

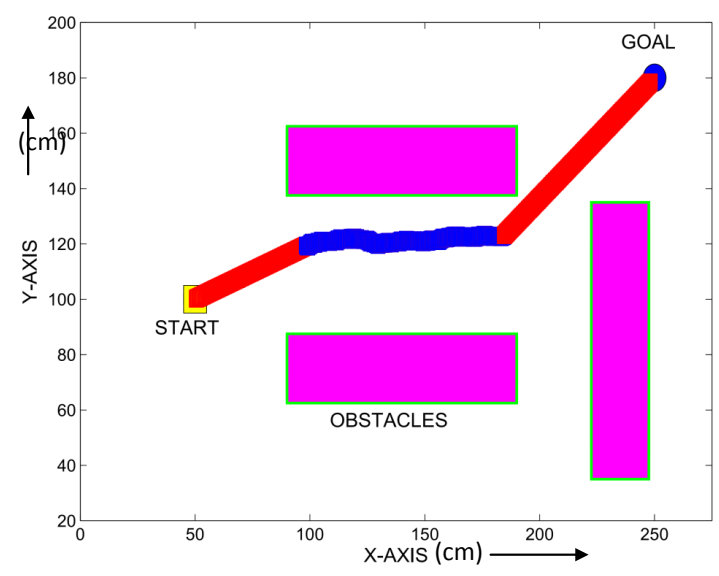

Figure 7: Mobile robot navigation in an unknown environment using CN-Fuzzy architecture

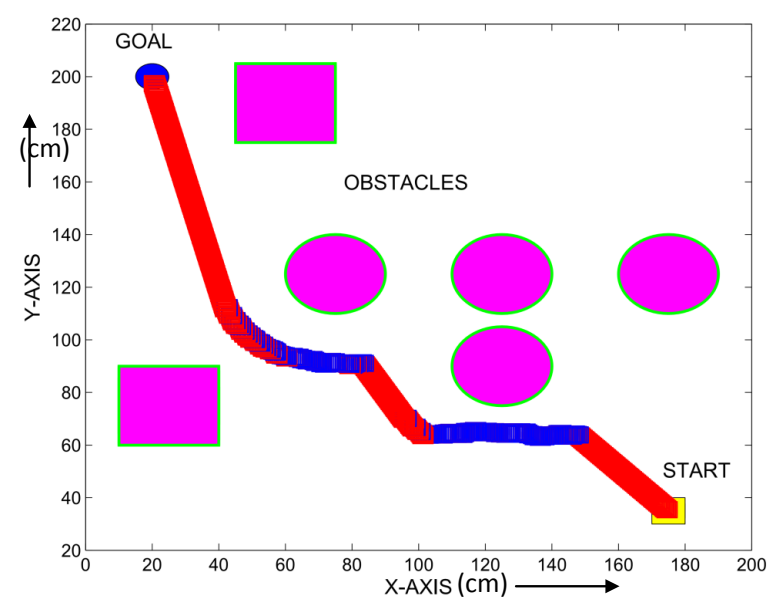

Figure 8: Mobile robot navigation in the cluttered environment using CN-Fuzzy architecture 
software on the HP $3.40 \mathrm{GHz}$ processor. Figure 5 illustrates the developed flowchart of mobile robot navigation and obstacle avoidance based on CN-Fuzzy architecture. Figures 6 to 9 shows the mobile robot navigation trajectories in the different static and dynamic environments. In the simulation results, it is assumed that the position of the start point and goal point are known. But the positions of all the obstacles in the environment are unknown for the robot. The dimension of the environments is $300 \mathrm{~cm}$ width and $300 \mathrm{~cm}$ height. A minimum threshold distance is fixed between the robot and the obstacle. Now if the robot detects the obstacle in the threshold range, then the proposed architecture estimates the desired turning direction of a mobile robot. Table 3 illustrates the navigation path length and time taken by the robot in the various unknown environments.

\section{Comparison With Previous Developed Techniques}

This section describes the computer simulation result comparisons between the previously developed techniques $[13,14]$ and proposed CN-Fuzzy architecture in the same environment.

\section{First Comparison with Developed Technique}

In article [13], the authors have designed goal-seeking, obstacle avoidance behavior, and other behavior for mobile robot navigation using fuzzy controller. Figures 10 and 11 illustrate the mobile robot navigation in the same environment without obstacle using fuzzy controller [13] and CN-Fuzzy architecture, respectively. From simulation result, it can be clearly seen that the robot covers shorter distance to reach the goal using proposed architecture as compared to previous technique [13]. Table 4 shows the path covered by the robot to reach the goal using fuzzy controller [13] and proposed CN-Fuzzy architecture. The centimetre measurements are taken on the proportional basis.

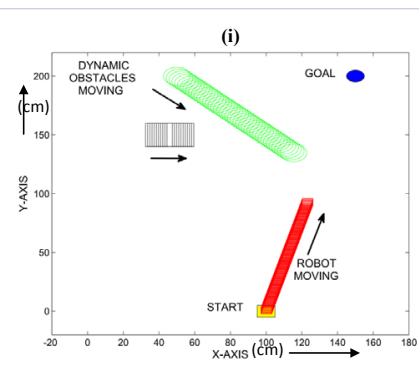

(iii)

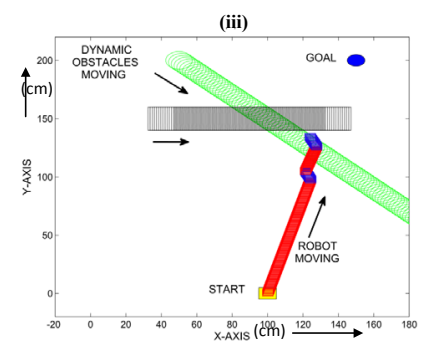

Figure 9: Mobile robot navigation in the dynamic environment using CN-Fuzzy architecture

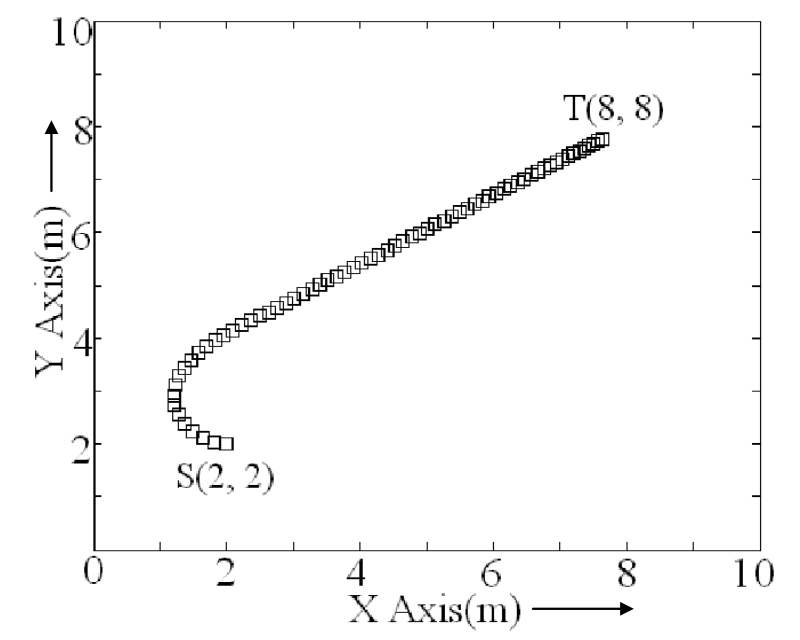

Figure 10: without Mobile robot navigation in an environment obstacle using fuzzy controller [13].

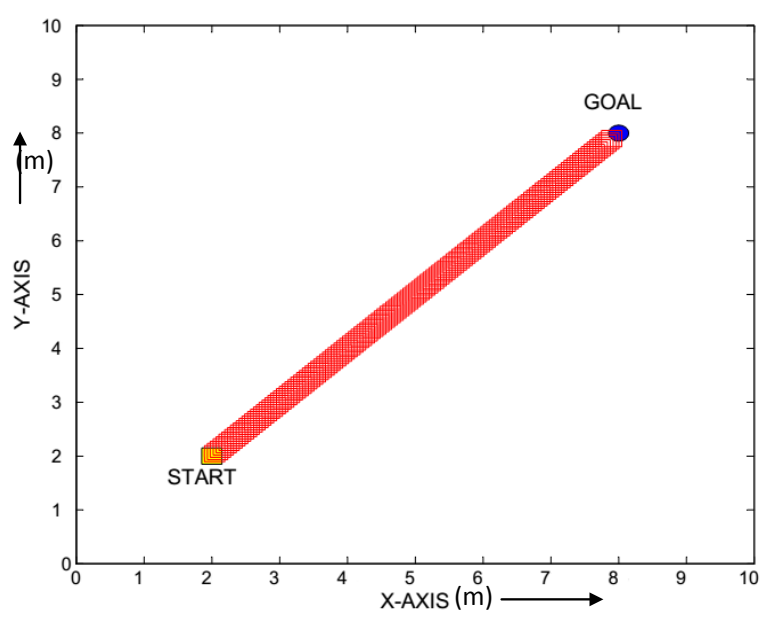

Figure 11: Mobile robot navigation in an environment without obstacle using CN-Fuzzy architecture

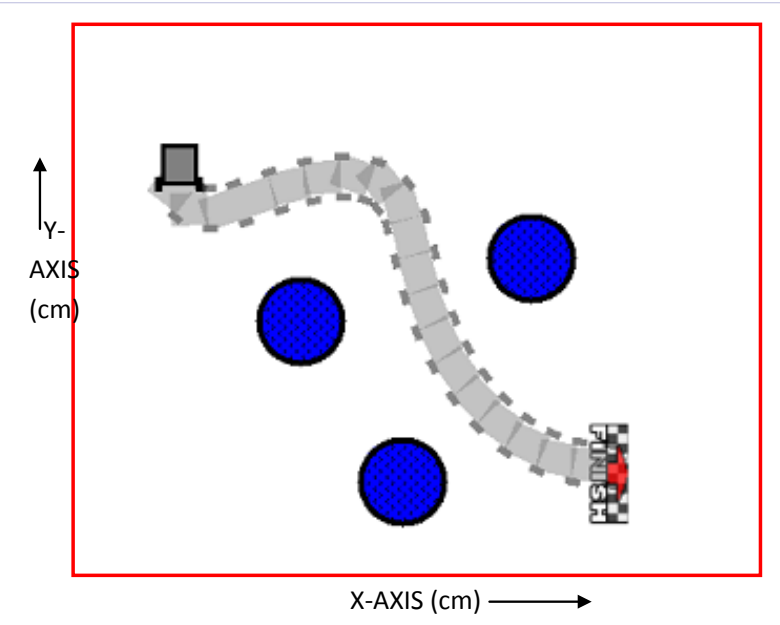

Figure 12: Mobile robot navigation in an environment with obstacles using artificial neural network 


\section{Second Comparison with Developed Technique}

In this section, the simulation result comparison has been made between the previous technique [14] and proposed CNFuzzy architecture in the same environment with the obstacles. In [14], the authors have discussed the motion and path planning of a car-like wheeled mobile robot between the stationary obstacles using backpropagation artificial neural network. Figure 12 shows the mobile robot navigation in an environment with obstacles using artificial neural network [14]. Figure 13 presents the path covered by the robot using proposed CN-Fuzzy architecture in the same environment. From the Figures 12 and 13, it is observed that the proposed architecture avoid the obstacles with some shorter distance or minimum steering as compared to previous model [14]. Table 5 illustrates the path traced (in $\mathrm{cm}$ ) by the robot to reach the goal using proposed architecture and previous model [14]. The centimetre measurements are taken on the proportional basis.

\section{Experimental Results}

\section{Experimental Mobile Robot Description}

This section describes the characteristic of the experimental mobile robot (Figure 14). The robot has two front wheels, which is powered by separate DC geared motors. The motor driver is used to control the velocity and direction of the robot. The width of the robot plate is $23 \mathrm{~cm}$, and the track width and height of robot are $30 \mathrm{~cm}$ and $8 \mathrm{~cm}$, respectively. The mobile robot is equipped with one sharp infrared range sensor on the front side, and the two ultrasonic range finder sensors fitted on the left and right side of the robot, as shown in Figure 15. Each sensor can read obstacle from $20 \mathrm{~cm}$ to $150 \mathrm{~cm}$ approximately. The minimum and maximum velocities of the experimental mobile robot are between $6.7 \mathrm{~cm} / \mathrm{sec}$ to $16.7 \mathrm{~cm} / \mathrm{sec}$ approximately.

\section{Experiments}

This section presents the experimental results of a mobile robot using CN-Fuzzy architecture in the different environments. The experiments have been performed by $\mathrm{C} / \mathrm{C}++$ running Arduino microcontroller based mobile robot. The proposed architecture controls the motor velocities (right and left) of the robot during navigation in the environment using sensor data interpretation. Figure 16 to 18 shows the real time navigation of the experimental mobile robot in the different environments. The width and height of the platform are $250 \mathrm{~cm}$ and $250 \mathrm{~cm}$, respectively. In

Table 3: Simulation results of mobile robot navigation in the different environments using CN-Fuzzy architecture

\begin{tabular}{|l|l|l|l|}
\hline Figure No. & Environment Type & $\begin{array}{l}\text { Travelling Path } \\
\text { Length (cm) }\end{array}$ & $\begin{array}{l}\text { Navigation } \\
\text { Time (sec) }\end{array}$ \\
\hline Figure 6 & Without obstacle & 103 & 11.6 \\
\hline Figure 7 & $\begin{array}{l}\text { Unknown } \\
\text { environment }\end{array}$ & 89 & 10.1 \\
\hline Figure 8 & $\begin{array}{l}\text { Cluttered } \\
\text { environment }\end{array}$ & 120 & 13.4 \\
\hline Figure 9 & $\begin{array}{l}\text { Dynamic } \\
\text { environment }\end{array}$ & 77 & 8.6 \\
\hline
\end{tabular}

Table 4: The simulation result comparison between the fuzzy controller [13] and proposed CN-Fuzzy architecture

\begin{tabular}{|l|l|l|}
\hline Figure No. & Method & $\begin{array}{l}\text { Navigation Path Length } \\
\text { (cm) }\end{array}$ \\
\hline Figure 10 & Fuzzy [13] & 51 \\
\hline Figure 11 & CN-Fuzzy architecture & 46 \\
\hline
\end{tabular}

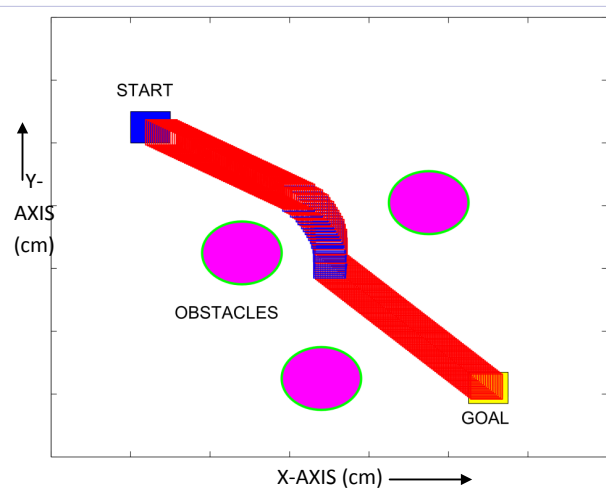

Figure 13: Mobile robot navigation in an environment with obstacles using CN-Fuzzy architecture

Table 5: The simulation result comparison between the artificial neural network [14] and proposed CN-Fuzzy architecture

\begin{tabular}{|l|l|l|}
\hline Figure No. & Method & $\begin{array}{l}\text { Navigation Path Length } \\
\text { (cm) }\end{array}$ \\
\hline Figure 12 & $\begin{array}{l}\text { Artificial neural network } \\
{[14]}\end{array}$ & 87 \\
\hline Figure 13 & CN-Fuzzy architecture & 80 \\
\hline
\end{tabular}

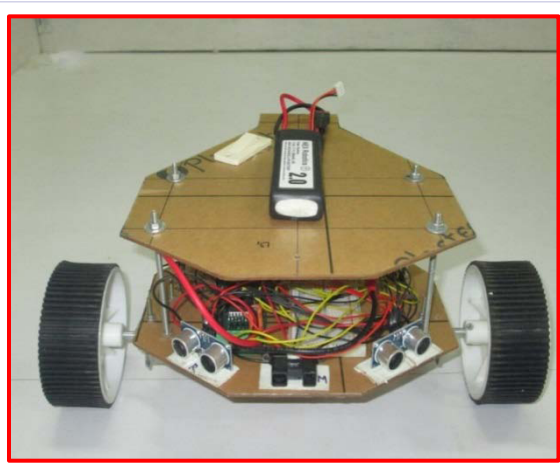

Figure 14: Experimental mobile robot

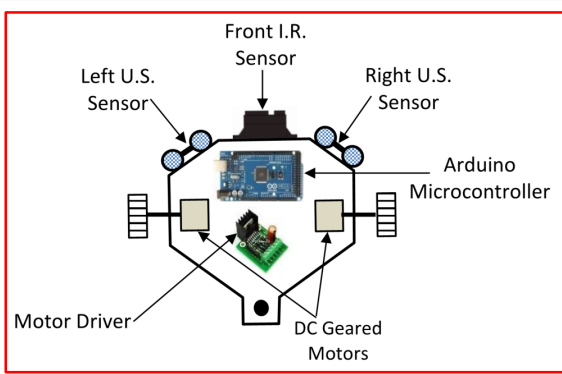

Figure 15: Sensor distribution of the experimental mobile robot 
the experimental results, it is assumed that the position of the start point and goal point are known. But the positions of all the obstacles in the environment are unknown for the robot. Firstly, the robot goes towards the goal in the environment, and if the sensor detects the obstacle in the threshold range, then the proposed architecture controls the velocity of the mobile robot. In Figure 17, the start position of the robot is $(50,100)$ $\mathrm{cm}$ and the position of the goal is $(250,180) \mathrm{cm}$. The starting angle between the robot and the goal is $21.8^{\circ}$. In Figure 18 , the start position of the robot is $(45,125) \mathrm{cm}$ and the goal position is $(130,40) \mathrm{cm}$. The starting angle between the robot and the goal is $45^{\circ}$. In Figures 17 and 18, if the left obstacle is near to the mobile robot, then the robot turns right, i.e. the velocity of the right motor is less than the velocity of left motor. Similarly, if the right obstacle is near to the mobile robot, then the robot turns left, i.e. the velocity of the right motor is more than the velocity of left motor. The average moving speed of the robot is $0.09 \mathrm{~m} /$ sec. The experimental results in the different snapshots verify

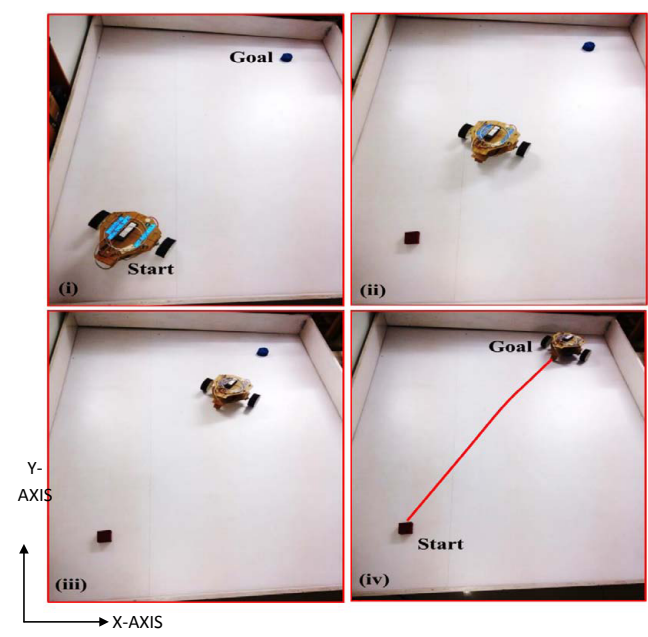

Figure 16: Experimental result of mobile robot navigation same as a simulation result (shown in Figure 6)

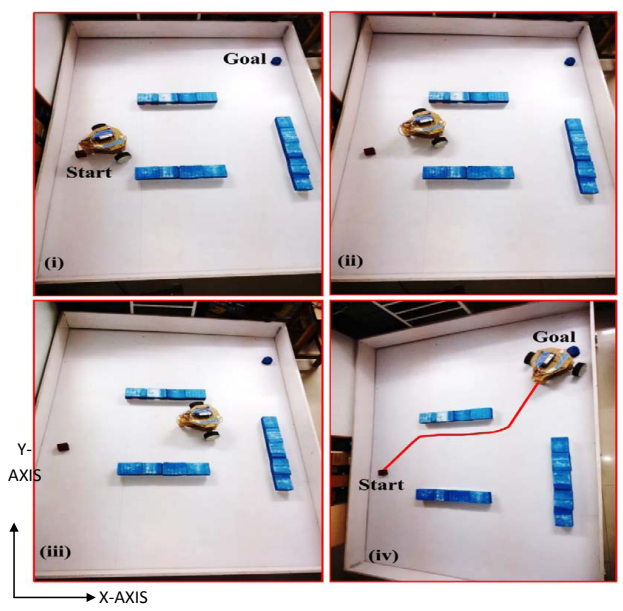

Figure 17: Experimental result of mobile robot navigation same as a simulation result (shown in Figure 7)

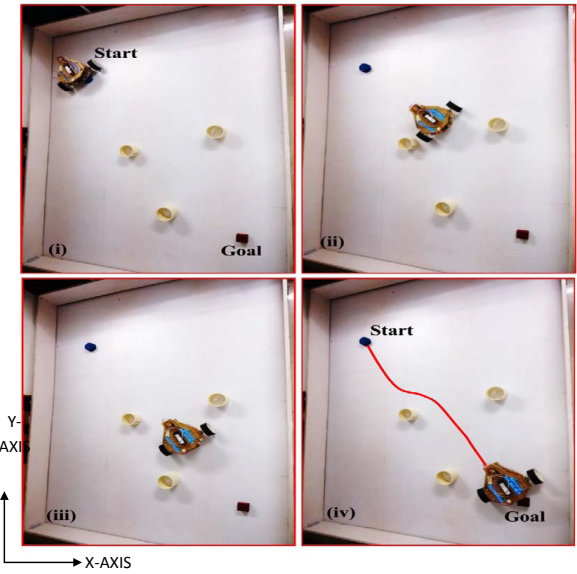

Figure 18: Experimental result of mobile robot navigation same as a simulation result (shown in Figure 13)

Table 6: Experimental results of a mobile robot navigation in the different environments using CN-Fuzzy architecture

\begin{tabular}{|l|l|l|l|}
\hline Figure No. & Environment Type & $\begin{array}{l}\text { Travelling Path } \\
\text { Length (cm) }\end{array}$ & $\begin{array}{l}\text { Navigation Time } \\
\text { (sec) }\end{array}$ \\
\hline Figure 16 & Without obstacle & 109 & 12.4 \\
\hline Figure 17 & $\begin{array}{l}\text { Unknown } \\
\text { environment }\end{array}$ & 94 & 10.8 \\
\hline Figure 18 & $\begin{array}{l}\text { Unknown } \\
\text { environment }\end{array}$ & 85 & 10.1 \\
\hline
\end{tabular}

Table 7: Travelling path lengths comparison between simulation and experimental results

\begin{tabular}{|c|c|c|c|}
\hline \multirow{2}{*}{$\begin{array}{l}\text { Figure No. } \\
\text { (Simulation and } \\
\text { Experimental res.) }\end{array}$} & \multicolumn{2}{|c|}{ Travelling Path Length $(\mathrm{cm})$} & \multirow{2}{*}{$\begin{array}{l}\text { Error between } \\
\text { simulation and } \\
\text { experimental } \\
\text { result }\end{array}$} \\
\hline & $\begin{array}{l}\text { Simulation } \\
\text { Result }\end{array}$ & $\begin{array}{l}\text { Experimental } \\
\text { Result }\end{array}$ & \\
\hline Figures 6 and 16 & 103 & 109 & $5.5 \%$ \\
\hline Figures 7 and 17 & 89 & 94 & $5.32 \%$ \\
\hline Figures 13 and 18 & 80 & 85 & $5.88 \%$ \\
\hline
\end{tabular}

Table 8: Navigation time comparison between simulation and experimental results

\begin{tabular}{|c|c|c|c|}
\hline \multirow{2}{*}{$\begin{array}{l}\text { Figure No. } \\
\text { (Simulation and } \\
\text { Experimental res.) }\end{array}$} & \multicolumn{2}{|c|}{ Navigation Time (sec) } & \multirow{2}{*}{$\begin{array}{l}\text { Error Between } \\
\text { Simulation and } \\
\text { Experimental } \\
\text { Result }\end{array}$} \\
\hline & $\begin{array}{l}\text { Simulation } \\
\text { Result }\end{array}$ & $\begin{array}{l}\text { Experimental } \\
\text { Result }\end{array}$ & \\
\hline Figures 6 and 16 & 11.6 & 12.4 & $6.45 \%$ \\
\hline Figures 7 and 17 & 10.1 & 10.8 & $6.48 \%$ \\
\hline Figures 13 and 18 & 9.4 & 10.1 & $6.93 \%$ \\
\hline
\end{tabular}

the effectiveness of the proposed architecture. Table 6 shows the real-time navigation path length and time taken by the robot in the various unknown environments. Tables 7 and 8 illustrate the travelling path length and navigation time comparison between the simulation and experimental results, respectively. In the comparison study between the simulation and experiments, it is observed that some errors have been found, these are happened due to slippage and friction during real time experiment. 


\section{Conclusion And Future Scope}

In this paper, the CN-Fuzzy architecture has been applied to the intelligent navigation of a mobile robot in unknown environments filled with obstacles. The major contributions of this present paper are summarized as follows: -

1) The cascade neural network is designed to train the robot to reach the goal in the environment. The inputs of cascade neural network are the obstacle distances, and the output is the turning angle between the robot and goal.

2) The fuzzy logic controller helps the robot to control the right motor velocity and left motor velocity in the environments for obstacle avoidance.

3) The proposed CN-Fuzzy architecture gives better results (in terms of path length) as compared to previous developed techniques [13] and [14], which proves the authenticity of the proposed architecture.

4) Moreover, the simulation and experimental results in the different environment show the effectiveness of the proposed architecture in the both static and dynamic environments. The average percentage of errors between simulation and experimental studies are found to be within $5.57 \%$ in terms of travelling path lengths and $6.62 \%$ in terms of navigation time.

The proposed techniques are developed and tested for the navigation of single robot in static and dynamic environments. In future research, this proposed architecture can be extended for multiple mobile robot navigation and obstacle avoidance.

\section{References}

1. Ma X, Li X, Qiao H. Fuzzy neural network-based real-time self-reaction of mobile robot in unknown environments. 2001;11 (8):1039-1052.

2. Godjevac J, Steele N. Neuro-Fuzzy control of a mobile robot. 1999;28(1):127-143.

3. Rai N, Rai B. Neural network based closed loop speed control of dc motor using Arduino UNO. 2013;4(2): 137-140.
4. Yang SX, Meng M. Neural network approaches to dynamic collisionfree trajectory generation. 200;31(3): 302-318.

5. Juang CF, Hsu CH. Reinforcement ant optimized fuzzy controller for mobile-robot wall-following control. 2009;56(10):3931-3940.

6. Algabri M, Mathkour H, Ramdane H, Alsulaiman M. Comparative study of soft computing techniques for mobile robot navigation in an unknown environment. 2015;50: 42-56.

7. Beom HR, Cho KS. A sensor-based navigation for a mobile robot using fuzzy logic and reinforcement learning. 1995;25(3):464-477.

8. Li W, Ma C, Wahl F M. A neuro-fuzzy system architecture for behavior-based control of a mobile robot in unknown environments. 1997;87(2):133-140.

9. Rossomando FG, Soria CM. Design and implementation of adaptive neural PID for nonlinear dynamics in mobile robots. 2015;13(4):913918.

10. Ming L, Zailin G, Shuzi Y. Mobile robot fuzzy control optimization using genetic algorithm. 1996;10(4):293-298.

11. Juang CF, Lai MG, Zeng WT. Evolutionary fuzzy control and navigation for two wheeled robots cooperatively carrying an object in unknown environments. 2015;45(9):1731-1743.

12. Chayjan RA, Ashari ME. Modeling Isosteric heat of soya bean for desorption energy estimation using neural network approach. 2010;70(4):616-625.

13. Qing-yong B, Shun-ming L, Wei-yan S, Mu-jin A. A fuzzy behavior-based architecture for mobile robot navigation in unknown environments. 2009:257-261.

14. Engedy I, Horvath G. Artificial neural network based local motion planning of a wheeled mobile robot. 2010:213-218.

15. Mohanty PK and Parhi DR. Navigation of autonomous mobile robot using adaptive network based fuzzy inference system. 2015;28(7):2861-2868.

16. Pothal JK and Parhi DR. Navigation of Multiple Mobile Robots in a Highly Clutter Terrains using Adaptive Neuro-Fuzzy Inference System. 2015;72:48-58.

17. Mamdani EH and Assilian S. An Experiment in Linguistic Synthesis with a Fuzzy Logic Controller. 1975;7(1):1-13. 